\title{
Manejo de neoplasias malignas gastrointestinales durante el embarazo
}

\author{
Eduardo Reyna-Villasmil. ${ }^{1}$
}

\begin{abstract}
RESUMEN
La incidencia de cáncer durante el embarazo es rara y con frecuencia es diagnosticado en etapas avanzadas. La frecuencia ha aumentado debido a la demora en la maternidad junto al aumento de tumores malignos dependientes de la edad entre los adultos jóvenes. Los cánceres más frecuentes diagnosticadas durante el embarazo son de mama, cuello uterino, hematológicas (linfomas y leucemias agudas) y melanoma. Los menos comunes son los gastrointestinales. El diagnóstico es un desafio, la sintomatología a menudo es atribuida a los síntomas del embarazo, lo que podría retrasar el diagnóstico y permitir que el cáncer progrese a estadios avanzados. Los médicos tratantes tienen dos problemas en estas embarazadas: la necesidad de tratamiento temprano del cáncer materno y el momento de interrupción del embarazo. Los médicos deben conocer los desafíos asociados al manejo de las embarazadas con esta condición y formular rápidamente planes terapéuticos de acuerdo a la edad gestacional.
\end{abstract}

Palabras clave: Tumores malignos, Sistema gastrointestinal, Cáncer, Manejo, Diagnóstico, Tratamiento.

Management of malignant gastrointestinal neoplasias during pregnancy

SUMMARY

The incidence of cancer during pregnancy is rare and is often diagnosed in advanced stages. The frequency has increased due to delayed motherhood along with the increase in age-dependent malignancies among young adults. The most common cancers diagnosed during pregnancy are breast, cervical, hematological (lymphomas and acute leukemias) and melanoma. The least common are gastrointestinal. Diagnosis is challenging, the symptomatology is often attributed to the symptoms of pregnancy, which could delay diagnosis and allow the cancer to progress to advanced stages. Treating physicians have two problems in these pregnant women: the need for early treatment of maternal cancer and the timing of termination of pregnancy. Physicians should be aware of the challenges associated with managing pregnant women with this condition and quickly formulate therapeutic plans according to gestational age.

Keywords: Malignant tumors, Gastrointestinal system, Cancer, Management, Diagnosis, Treatment.

\section{INTRODUCCIÓN}

El número de casos de cáncer en embarazadas es de aproximadamente 1 por cada 1000 embarazos, por lo tanto, es casi imposible obtener resultados de ensayos clínicos que puedan aclarar las dudas. El conocimiento

\footnotetext{
'Doctor en Medicina Clínica. Especialista en Ginecología y Obstetricia. Servicio de Obstetricia y Ginecología. Hospital Central "Dr. Urquinaona". Maracaibo. Estado Zulia. Venezuela.
}

Forma de citar este artículo: Reyna-Villasmil E. Manejo de neoplasias malignas gastrointestinales durante el embarazo. Rev Obstet Ginecol Venez. 2021; 81(3):273286. https://doi.org/10.51288/00810311 existente ha sido obtenido de estudios retrospectivos, informes o series de casos y experiencia clínica (1). Los datos de neoplasias gastrointestinales que ocurren durante el embarazo son escasos. El cáncer colorrectal ocurre en 2 de cada 100000 embarazos, el cáncer gástrico en $0,1 \%$ de la población japonesa $\mathrm{y}$, probablemente, con menor frecuencia en otras poblaciones y solo existen informes de casos aislados de cáncer de páncreas (2-5).

En las embarazadas, las neoplasias gastrointestinales pueden manifestarse con dolor abdominal, náuseas y vómitos, melena, hematoquecia, anemia, dolor 
lumbar y diarrea. Algunos de estos síntomas pueden asociarse al embarazo, lo que contribuye al retraso diagnóstico. Las pruebas de diagnóstico durante el embarazo incluyen: ecografía (segura tanto para la embarazada como para el feto), resonancia magnética (segura para la madre y el feto, pero no debe utilizarse durante el primer trimestre del embarazo), endoscopia digestiva alta y sigmoidoscopia (seguras, pero deben realizarse solo ante las sospechas de neoplasias gastrointestinales), así como biopsia por aspiración y resección (procedimientos considerados seguros durante el embarazo) (3).

En la mayoría de los casos, la neoplasia maligna es diagnosticada en etapas avanzadas y, generalmente, durante el primer trimestre del embarazo. La estrategia terapéutica en la mayoría de los casos está basada en la interrupción del embarazo, en el retraso del tratamiento hasta el parto o el parto pretérmino iatrogénico. En la mayoría de los tumores, la tasa de mortalidad es cercana al $50 \%(5)$.

Estudios retrospectivos de neoplasias gastrointestinales diagnosticadas (cáncer rectal, colorrectal, hepático, duodenal y sarcoma de Ewing) antes o durante el embarazo, en 20 años, identificaron que los signos clínicos más comunes incluyen: náuseas, rectorragia, dolor abdominal, vómitos y aumento de la circunferencia abdominal. Las embarazadas deben ser sometidas a endoscopia con recolección de muestras para biopsia, resección tumoral y, en algunos casos, sometidas a quimioterapia. En la mayoría de los casos el tratamiento oncológico debe ser administrado después del parto dependiendo del tipo de cáncer (6).

El objetivo de la revisión es analizar el manejo de las neoplasias malignas gastrointestinales durante el embarazo.

\section{CÁNCER DE ESÓFAGO}

La coexistencia de cáncer de esófago y embarazo es extremadamente rara, con solo pocos casos descritos en la literatura. Uno de los casos reportados era de una embarazada con disfagia, hematemesis y pérdida de peso en el tercer trimestre (7). El diagnóstico, por endoscopia y resonancia magnética, demostró el tumor del esófago inferior, que llegaba hasta el cardias. Debido a la inmadurez pulmonar fetal, se decidió conducta expectante y administración de corticosteroides. A las 34 semanas, el embarazo fue interrumpido. Dos semanas después del parto, la paciente fue sometida a toracotomía, la cual demostró que el tumor no era resecable (lesión localmente avanzada con ganglios linfáticos y metástasis hepáticas) (8).

Otro caso de cáncer de esófago fue descrito en una embarazada que presentó disfagia y pérdida de peso a las 27 semanas. El examen endoscópico y la tomografía computarizada del tórax demostraron cáncer localmente avanzado del esófago medio. Debido al embarazo, la paciente no podía ser tratada con quimio-radioterapia neoadyuvante, pero fue sometida a cirugía, siendo posteriormente tratada en forma concomitante con progesterona y esteroides. A las 28 semanas de gestación, se realizó esofagectomía y esofagogastrostomía. El periodo de hospitalización fue complicado por paro cardiaco en el posoperatorio tardío (35 días después de la cirugía) (9).

El manejo de los casos reportados es diferente y ajustado a cada paciente en forma individual, según la edad gestacional y la experiencia del médico tratante. Para el diagnóstico de neoplasias esofágicas, tanto el examen endoscópico como la resonancia magnética parecen ser seguros, mientras que la tomografía computada debe evitarse debido al riesgo de defectos fetales. El diagnóstico, generalmente, es realizado 
en etapas avanzadas en las cuales es imposible el tratamiento radical. En el manejo inicial se debe valorar la madurez pulmonar fetal. El tratamiento sintomático debe ser específico y óptimo, mientras que la radiación, quimioterapia o cirugía debe realizarse inmediatamente después del parto. El tratamiento quirúrgico se puede realizar durante el embarazo, pero está asociado con gran número de complicaciones posoperatorias. Si no es posible continuar con el embarazo, se debe interrumpir e iniciar el tratamiento de manera inmediata (figura 1) $(8,9)$.

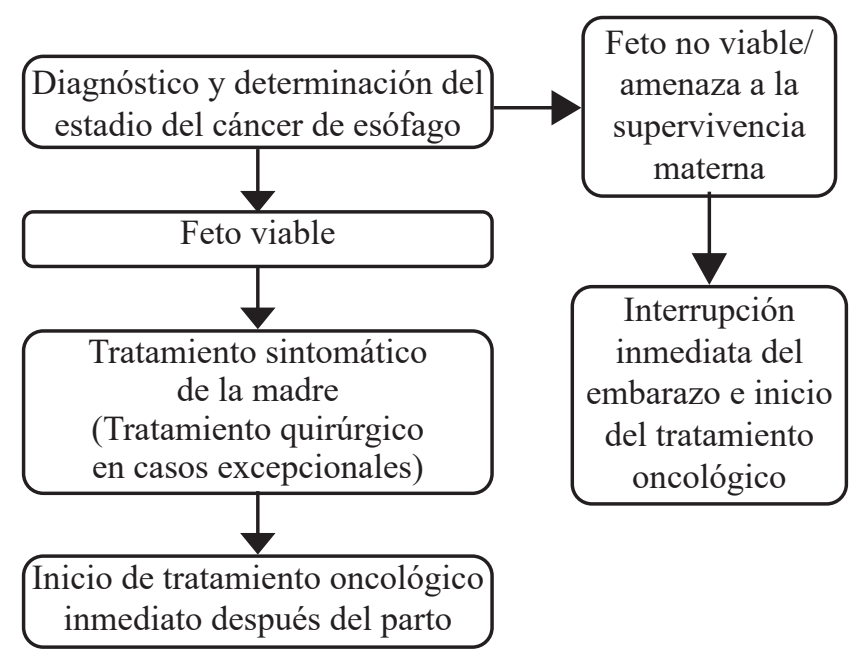

Figura 1. Diagrama de manejo en embarazadas con cáncer de esófago

\section{CÁNCER DE ESTÓMAGO}

El cáncer de estómago durante el embarazo es raro y esta descrito principalmente en mujeres de origen oriental. Al igual que el cáncer de esófago, a menudo el diagnóstico es realizado en etapas avanzadas. La definición incluye los casos diagnosticados durante el embarazo o hasta un año después del parto y su incidencia es aproximadamente de $0,1 \%$ de todos los casos de cáncer gástrico (10). En un estudio retrospectivo, su frecuencia fue de $0,103 \%$ (11). Los síntomas más comunes son náuseas y vómitos, que también ocurren durante el embarazo. La mayoría de los casos tenía malignidad histológica alta, baja diferenciación tumoral y tipo difuso.

La mayoría de las pacientes fue diagnosticada con cáncer en etapas localmente avanzadas o enfermedad generalizada. Solo una cuarta parte de los casos fue sometida a tratamiento quirúrgico radical. La supervivencia a los tres años fue de $23 \%$, comparada con $52 \%$ en el grupo control (mujeres en edad fértil, entre 20 y 40 años con cáncer de estómago no relacionado con el embarazo). El análisis multivariante identificó dos factores pronósticos independientes: metástasis de ganglios linfáticos y tipo de tratamiento quirúrgico. Los autores recomiendan el tratamiento inmediato en estos casos, incluida la interrupción del embarazo, y solo en casos de cáncer en estadios tempranos puede retrasarse el tratamiento hasta alcanzar la madurez pulmonar fetal (11).

En algunos casos justificados, en etapas en las que es imposible la curación, y después de discutir la situación con la paciente, puede realizarse solo tratamiento sintomático hasta alcanzar la madurez fetal. Existe un informe de caso de una embarazada que fue diagnosticada con cáncer de estómago a las 28 semanas y cuyo primer síntoma fue taponamiento cardiaco y líquido en cavidades pleurales. En este caso, el tratamiento fue conservador (pericardiocentesis y pleurocentesis) hasta alcanzar la madurez pulmonar fetal. El embarazo fue interrumpido a las 30 semanas debido a compromiso fetal. Esta experiencia sugiere que en casos de diagnóstico antes de las 24 semanas de embarazo, la conducta debe ser interrupción inmediata del embarazo y tratamiento quirúrgico, pero si el diagnóstico es entre las 24 y 29 semanas, el tratamiento depende de la resecabilidad de la lesión y, en el caso de poder ser extirpada, la cirugía debe ser la primera opción, independientemente de los riesgos fetales. Luego de las 29 semanas se debe posponer la cirugía hasta después del parto $(12,13)$. Es importante 
recordar que el cáncer en fase de diseminación puede producir metástasis a placenta y útero (14).

Otro síntoma del cáncer gástrico que coexiste con el embarazo puede ser el nódulo de la hermana María José o nódulo umbilical. Su presencia sugiere la propagación al peritoneo y generalización de la enfermedad (15). Existe un caso reportado de este signo durante el embarazo, el cual fue interrumpido para realizar citorreducción tumoral máxima (gastrectomía, esplenectomía, colecistectomía, hemicolectomía derecha, ooforosalpingectomía bilateral y extirpación de la cicatriz umbilical). Después del parto, comenzó inmediatamente la quimioterapia, que fue interrumpida por aparición de abscesos abdominales y peritonitis. Este caso demuestra que el diagnóstico de cáncer de estómago en embarazadas es tardío y que no existen síntomas hasta en $80 \%$ de los casos (16-19). La tasa de supervivencia anual y a los 2 años es de $18,0 \%$ y $15,1 \%$, respectivamente. Solo 5 pacientes sobrevivieron más de 3 años sin recurrencias (20).
Los datos sobre el uso de quimioterapia en embarazadas con cáncer gástrico son muy limitados y provienen de informes de casos únicos. Por lo tanto, la decisión de comenzar el tratamiento sistémico debe tomarse con precaución. En el primer trimestre del embarazo no está recomendada, debido al riesgo de aborto espontáneo o muerte fetal. En contraste, después del segundo trimestre es relativamente segura y el porcentaje de defectos congénitos marcados no difiere significativamente, comparado con la embarazadas sanas $(18,19)$. Actualmente no existe un régimen de quimioterapia estándar para embarazadas con cáncer de estómago. Los datos de la literatura incluyen los siguientes fármacos: docetaxel, paclitaxel (generalmente en régimen semanal) y 5-fluoracilo (principalmente en pacientes con enfermedad avanzada y metástasis a distancia, que es potencialmente mortal para la madre y el feto) (figura 2) (18).

El manejo de las embarazadas con cáncer de estómago depende de la edad gestacional al momento del

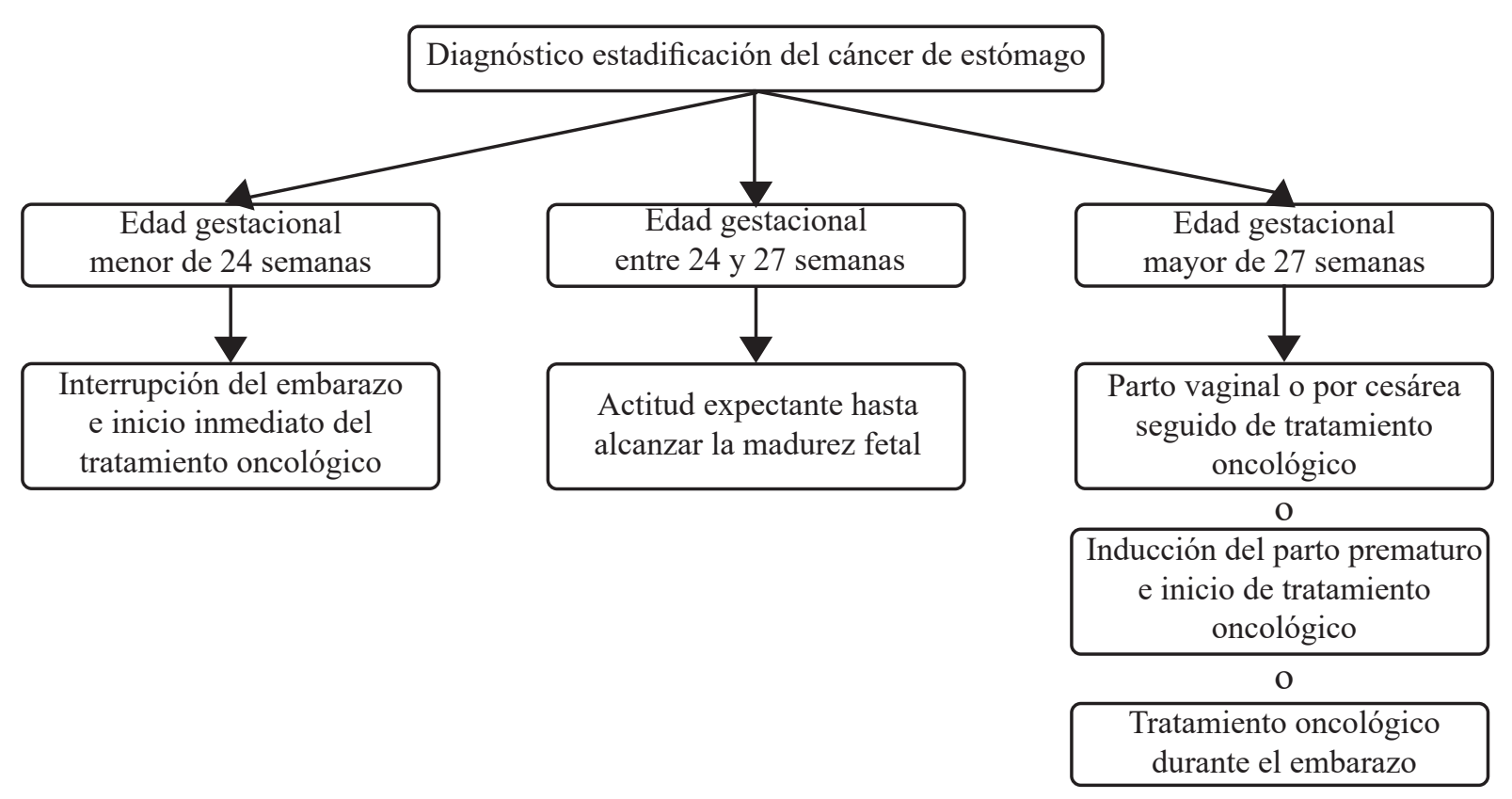

Figura 2. Diagrama de manejo de embarazadas con cáncer de estómago 
diagnóstico, estadio del cáncer y estado general de la paciente. La mayoría de los casos son diagnosticados en etapas avanzadas, lo que impide el tratamiento oncológico radical y es una amenaza para la madre y el feto. Sobre estos hallazgos, parece que el manejo óptimo antes de las 24 semanas de embarazo es la interrupción del embarazo y comenzar inmediatamente el tratamiento oncológico (cirugía o quimioterapia sistémica). Entre las 24 y 27 semanas es posible mantener una actitud expectante hasta alcanzar la madurez pulmonar fetal, especialmente en aquellos casos de cáncer temprano. Luego de las 27 semanas, es recomendable interrumpir el embarazo de forma inmediata para iniciar el tratamiento oncológico.

\section{PSEUDOMIXOMA PERITONEAL}

El pseudomixoma peritoneal es un tumor extremadamente raro (2 casos por cada $10 \quad 000$ laparotomías) y es 3 veces más frecuente en mujeres que en hombres. Esta caracterizado por presencia de tumor mucoso benigno o maligno, generalmente de origen apendicular u ovárico, que produce secreción mucosa gelatinosa intraperitoneal $(21,22)$. Hasta la fecha, solo se han descrito algunos casos aislados en embarazadas.

Entre los informes de casos citados está el de una paciente con embarazo de 17 semanas y diagnóstico ecográfico de tumor de ovario derecho. Durante la cirugía se encontró un tumor ovárico mucogénico, causante del pseudomixoma peritoneal. El embarazo fue interrumpido a las 35 semanas. Posteriormente, la paciente fue sometida a cirugía citorreductora óptima combinada con quimioterapia hipertérmica intraperitoneal, que fueron realizadas 3 semanas después del parto, y luego fue sometida a quimioterapia sistémica. La paciente no presentó recaídas durante los 5 años de seguimiento (23).

Existe otro informe de caso de pseudomixoma peritoneal secundario a cáncer colorrectal, que se diagnosticó a las 24 semanas de embarazo. Debido a la sospecha clínica, la paciente fue sometida a laparotomía exploradora encontrándose gran cantidad de líquido ascítico gelatinoso y amarillento. Se tomó la decisión de interrumpir el embarazo a las 34 semanas. La paciente fue operada nuevamente, realizándose resección de lesiones peritoneales, apendicetomía y resección parcial del colon transverso, seguida de quimioterapia sistémica. Seis meses después del tratamiento, y a pesar de la quimioterapia, la paciente murió (24).

El manejo del pseudomixoma peritoneal durante el embarazo depende de la edad gestacional, la agresividad de la lesión primaria y el compromiso maternofetal. Si el diagnóstico es realizado en el primer o segundo trimestre y se confirma la naturaleza agresiva del tumor primario, el retraso en el tratamiento está asociado con complicaciones maternas, por lo tanto, se debe considerar la interrupción del embarazo. Durante el tercer trimestre es necesario posponer el tratamiento oncológico hasta después del parto (en forma ideal, a las 35 semanas). Si el tipo del tumor es de bajo o moderado grado, es recomendable retrasar el tratamiento después del parto a las 35 semanas o hasta la fecha planificada para este (figura 3) (23-25).

En el segundo y tercer trimestre del embarazo, el tratamiento quirúrgico está asociado con bajo riesgo de aborto espontáneo o parto pretérmino, por lo que la cirugía puede realizarse como procedimiento de diagnóstico o terapéutico, especialmente en pacientes con síntomas abdominales agudos. Otras pruebas de diagnóstico (resonancia magnética y endoscopia) parecen seguras después del primer trimestre del embarazo. El tratamiento quirúrgico final debe posponerse hasta la interrupción del embarazo. La cirugía citorreductora extendida (óptima) y la quimioterapia hipertérmica intraperitoneal están contraindicadas en embarazadas. En aquellos casos 


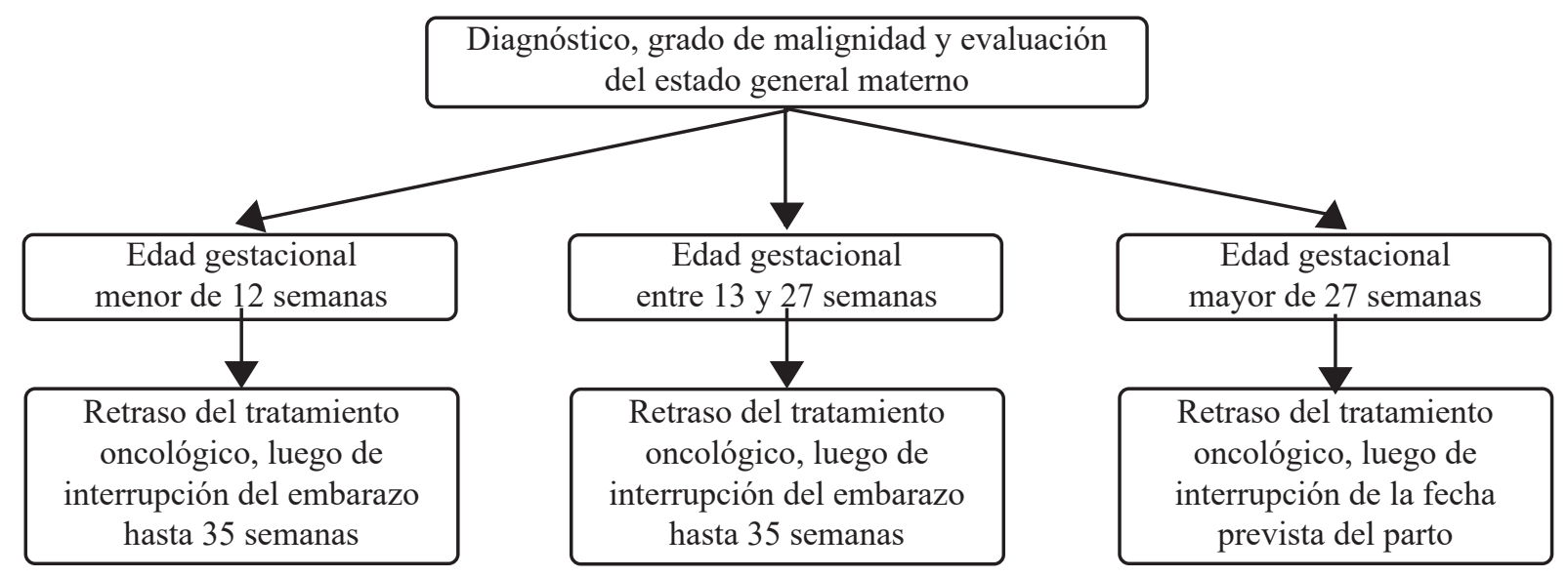

Parto vaginal o por cesárea solo por razones obstétricas. El tratamiento oncológico planificado consiste en cirugía citorreductora óptima, quimioterapia hipertérmica intraperitoneal y posiblemente quimioterapia sistémica

Figura 3. Diagrama de manejo de embarazadas con pseudomixoma peritoneal de bajo o moderado grado

de absoluta necesidad, la quimioterapia sistémica durante el segundo o tercer trimestre del embarazo es aceptable. El método de interrupción del embarazo es importante, la cesárea está contraindicada debido al alto riesgo de implantación de células malignas en abdomen y pared abdominal, por lo tanto, solo debe realizarse por indicaciones obstétricas (23-25). Debido a la muy baja incidencia de esta condición durante el embarazo, parece que el tratamiento también puede realizarse en pacientes con pseudomixoma peritoneal de origen no apendicular (figura 4).

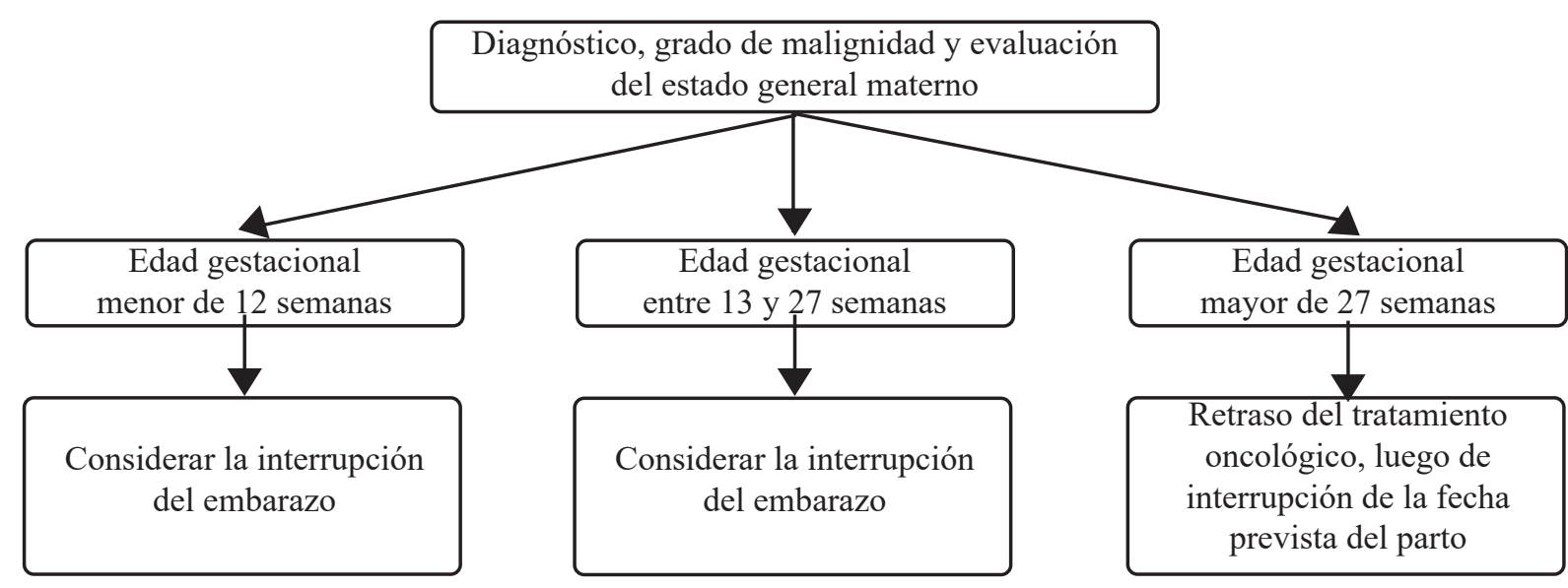

Parto vaginal o por cesárea solo por razones obstétricas. El tratamiento oncológico planificado consiste en cirugía citorreductora óptima, quimioterapia hipertérmica intraperitoneal y posiblemente quimioterapia sistémica

Figura 4. Diagrama de manejo de embarazadas con pseudomixoma peritoneal de alto grado 


\section{CÁNCER COLORRECTAL}

El cáncer colorrectal es poco frecuente en embarazadas (26). La frecuencia estimada varía de 1 caso por 13000 a 100000 embarazos $(0,002 \%)(27,28)$. La localización más común en embarazadas es el recto $(64 \%-86 \%)$ y generalmente es diagnosticado en estadio clínico III (60\%) en el segundo o tercer trimestre (29).

Hasta la fecha, existen varios estudios retrospectivos que evalúan el manejo del cáncer colorrectal en embarazadas. Un análisis retrospectivo de casos de cáncer colorrectal durante el embarazo demostró que la mayoría de los casos fue diagnosticada después del parto. Al compararlo con el grupo control, presentaron una incidencia significativamente mayor de cesárea e infecciones puerperales. No se encontraron diferencias en la supervivencia general entre ambos grupos (30). Otro estudio evaluó la resultante en embarazadas después del tratamiento y demostró un riesgo significativamente mayor de hemorragia prenatal, hemorragia posparto, cesárea, baja puntuación de Apgar, necesidad de reanimación neonatal y de atención especial después del ingreso hospitalario. También encontró que la cirugía estaba asociada a mayor riesgo y hospitalización posnatal prolongada. No encontraron diferencias significativas sobre la tasa de muertes perinatales. Por otra parte, el diagnóstico de cáncer rectal, comparado con el de colon, estaba asociado de forma independiente con aumento significativo de efectos adversos tanto en la madre como en el recién nacido. Además, los resultados indican una frecuencia mayor de complicaciones después del tratamiento (31).

Al igual que en otros tipos de cáncer, el manejo de las embarazadas con cáncer colorrectal depende de la ubicación (recto o colon), estadio y riesgos maternofetales. Para establecer el diagnóstico y estadio del tumor, se pueden utilizar endoscopia, ecografía y resonancia magnética (no es recomendable su uso en el primer trimestre del embarazo). El curso de acción adicional depende de la edad gestacional. En pacientes con cáncer de colon diagnosticado en la primera mitad del embarazo (antes de las 20 semanas), la cirugía puede realizarse en forma segura, especialmente si es urgente (figura 5). Dependiendo del resultado histopatológico

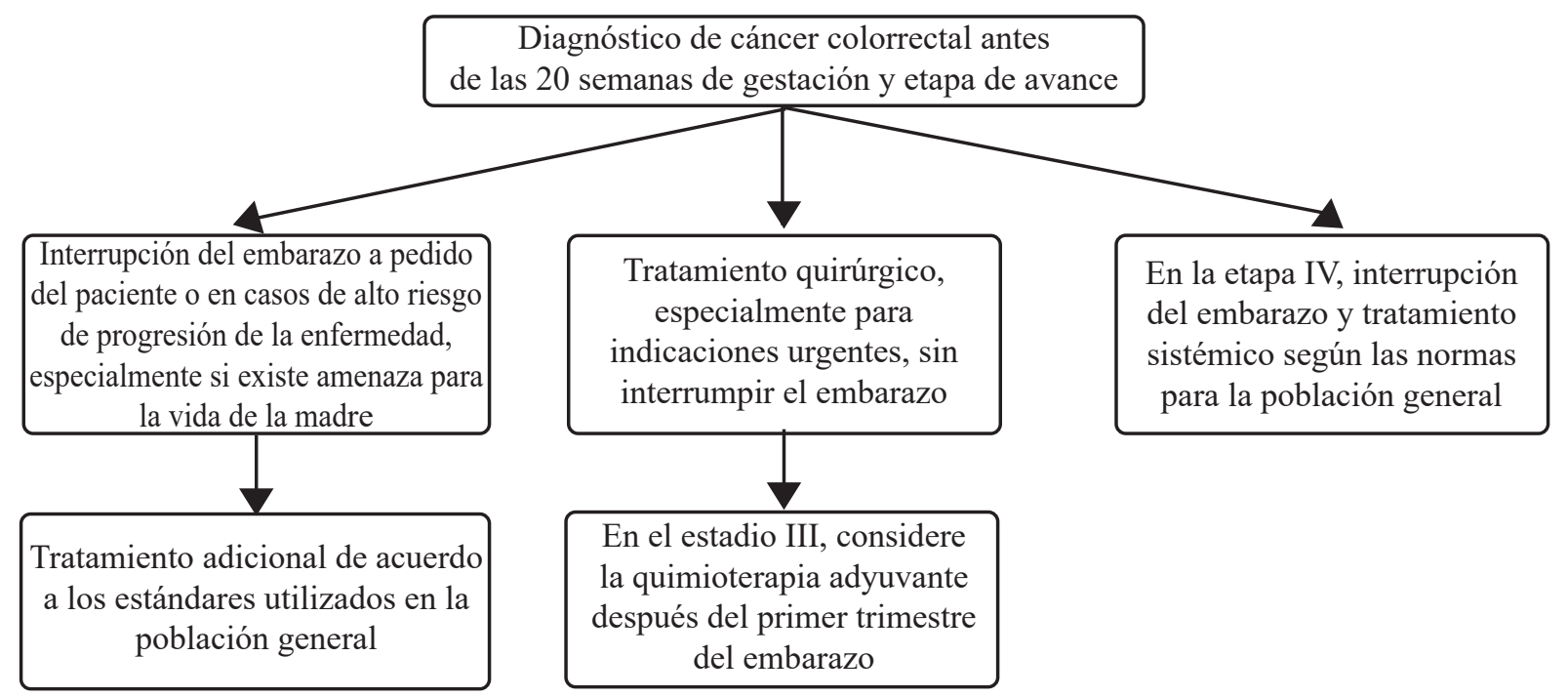

Figura 5. Diagrama de manejo para embarazadas con cáncer colorrectal antes de 20 semanas. 
posoperatorio y la gravedad, se establece un manejo adicional (32):

- Estadio I o II, realizar seguimiento y parto espontaneo,

- Estadio III: quimioterapia complementaria (después del primer trimestre del embarazo) o seguimiento e interrupción del embarazo.

- Estadio IV: interrupción del embarazo y quimioterapia paliativa.

En casos de diagnóstico en la segunda mitad del embarazo (después de las 20 semanas) es aconsejable el retraso del tratamiento hasta alcanzar la madurez pulmonar fetal y, después del parto, realizar el tratamiento de acuerdo a los estándares para pacientes no embarazadas. En situaciones excepcionales, por ejemplo, riesgo de obstrucción intestinal, se puede realizar la cirugía antes del parto, mientras que la quimioterapia adyuvante en el estadio III solo se puede utilizar después de la interrupción del embarazo. Si se planea realizar la cesárea, es posible también proceder a la resección del tumor durante el procedimiento.
No obstante, la mejor alternativa es realizar la cirugía varias semanas después del parto, cuando el útero ha involucionado y disminuye el edema vascular (figura 6) (32).

En casos de cáncer rectal, el procedimiento es similar al tratamiento del cáncer de colon. Si el diagnóstico se realiza antes de las 20 semanas de embarazo, retrasar el tratamiento puede conducir a progresión de la enfermedad y mayor riesgo de metástasis. Por lo tanto, la interrupción del embarazo y el tratamiento deben considerarse de acuerdo a los estándares de la población general. La cirugía puede realizarse sin interrumpir el embarazo y usar quimioterapia después del primer trimestre. El uso de radioterapia está completamente contraindicado debido al alto riesgo de alteraciones fetales. En la segunda mitad del embarazo, el tratamiento oncológico puede diferirse hasta alcanzar la madurez pulmonar fetal y, luego de la interrupción del embarazo, realizarse de acuerdo con los estándares para pacientes no embarazadas. Al igual que en pacientes con cáncer de colon, en caso de cesárea, la resección puede realizarse durante el procedimiento (32-35).

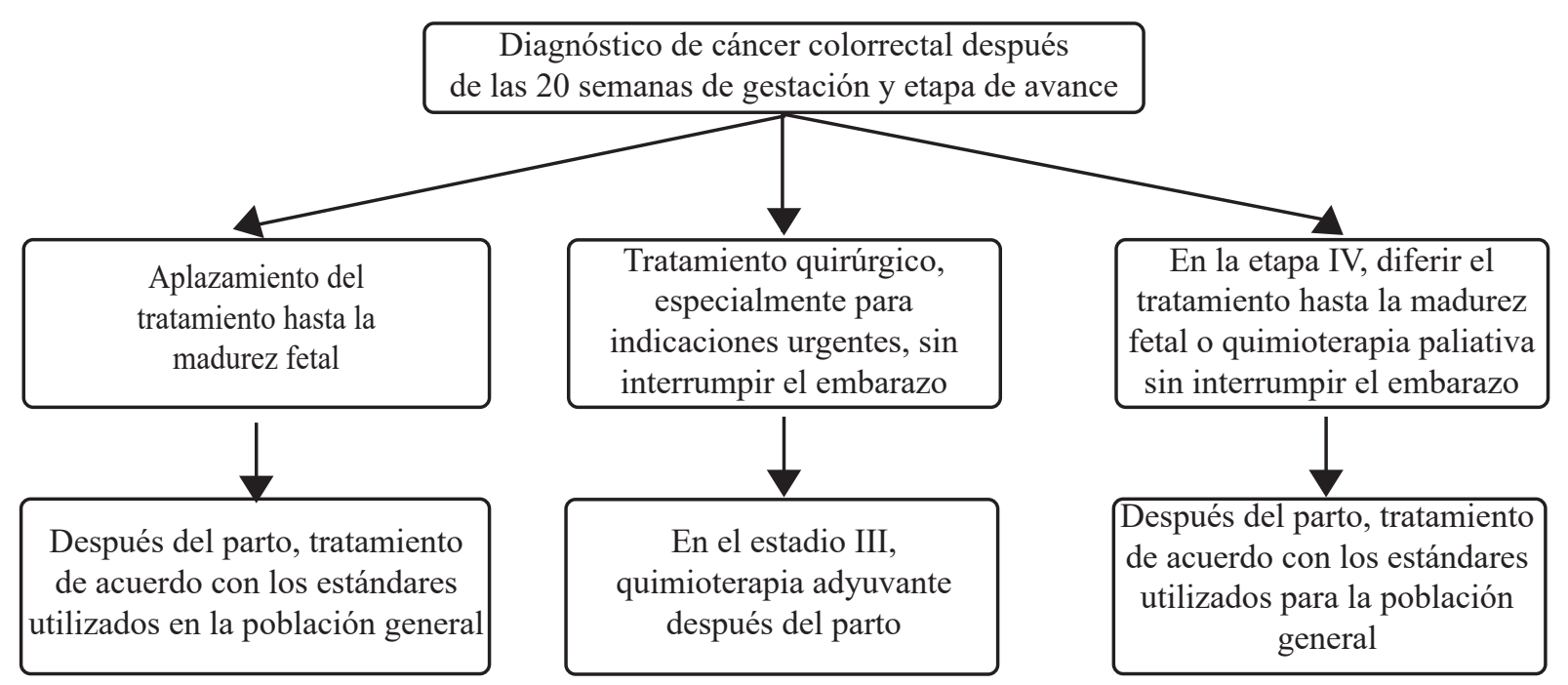

Figura 6. Diagrama de manejo para embarazadas con cáncer colorrectal después de 20 semanas. 
Los datos del uso de quimioterapia durante el embarazo para el tratamiento del cáncer colorrectal son limitados. No existen observaciones a largo plazo de los niños sometidos a quimioterapia durante la vida intrauterina. En los casos de estadio IV en embarazadas, es posible considerar el tratamiento sistémico, especialmente en caso de tumores muy agresivos. Durante el primer trimestre del embarazo, debido al desarrollo fetal, la quimioterapia está absolutamente contraindicada y solo puede usarse después de la interrupción del embarazo. Actualmente no existe un régimen de quimioterapia estándar para embarazadas con este tipo de cáncer (36-39). Algunos autores no recomiendan el uso de oxaliplatino durante el embarazo debido al potencial riesgo de neurotoxicidad y sus efectos negativos sobre el desarrollo cerebral fetal. Tampoco es recomendable usar bevacizumab debido al riesgo de alteración de la perfusión placentaria. No existen datos suficientes sobre la seguridad del uso de cetuximab y panitumumab en embarazadas $(40,41)$.

\section{OTROS TUMORES MALIGNOS GASTROINTESTINALES}

\section{Tumores pancreáticos}

Hasta la fecha, solo se han descrito en la literatura algunos casos de tumores pancreáticos durante el embarazo. En la población general, constituye $5 \%$ de todos los casos de cáncer, ocurre principalmente en mujeres y con mayor frecuencia en la región de la cola del páncreas. El estándar de tratamiento de lesiones sin signos de malignidad es la pancreatectomía distal. Durante el embarazo, la recomendación es realizar la cirugía durante el primer trimestre, ya que se considera seguro. Las indicaciones de cirugía son: histología potencialmente maligna, riesgo de agravamiento de la sintomatología asociado de crecimiento tumoral rápido y repentino, ruptura del tumor durante el parto, posibilidad de alteraciones del crecimiento intrauterino del feto y dependencia hormonal del tumor (42). El tratamiento quirúrgico solo puede posponerse si existe alto riesgo de complicaciones maternofetales asociadas a la cirugía y es recomendable la interrupción del embarazo por cesárea (42-44).

El manejo de los tumores de islotes pancreáticos es diferente. Debido al pequeño porcentaje de lesiones malignas (90\% de los tumores son benignos), es preferible tratamiento conservador y la cirugía solo debería realizarse después del parto (45).

Tumor carcinoide apendicular

El tumor carcinoide pertenece a los tumores neuroendocrinos de bajo grado que pueden secretar serotonina. Constituye $2 \%$ de todos los tumores malignos intestinales y $85 \%$ de los tumores malignos apendiculares. Es posible detectarlo en $0,16 \%-0,7 \%$ de todas las apendicetomías (46). Existen informes de algunos pocos casos descritos en embarazadas (47).

El pronóstico depende de varios factores: tamaño del tumor, ubicación anatómica en el apéndice cecal, presencia de metástasis y síntomas del síndrome carcinoide. El factor más importante es el tamaño del tumor (valor de corte 2 centímetros), el cual está asociado con mayor riesgo de metástasis a ganglios linfáticos. Cerca del $75 \%$ de los tumores se encuentran en la parte inferior, $20 \%$ en la porción central y $5 \%$ en la base del apéndice. Generalmente, es diagnosticado de forma accidental, después de una cirugía por otras razones y, en ocasiones, durante la cesárea (46-50).

Uno de los signos clínicos es el dolor abdominal agudo inespecífico, sin causa aparente al examen físico e investigación básica. En la ecografía, es posible observar líquido libre en cavidad abdominal, hiperemia y aumento de volumen del apéndice, que puede ser similar a la apendicitis aguda. El diagnóstico final está basado en la histopatología posoperatoria. Las 
pruebas diagnósticas útiles incluyen: concentraciones urinarias de serotonina, ácido 5-hidroxiindolacético y concentraciones séricas elevadas de cromogranina A. La gammagrafía con octreótide (análogo radiomarcado de somatostatina) no está recomendada durante el embarazo (47-50).

En la embarazada con enfermedad carcinoide, el tratamiento depende de la edad gestacional y grado de malignidad del tumor. La apendicetomía durante el segundo trimestre del embarazo es segura y el manejo posoperatorio depende de los hallazgos histopatológicos. Si la variedad histológica es agresiva, el tratamiento de elección es la hemicolectomía, que debe realizarse después de la interrupción del embarazo. Una posible alternativa al tratamiento quirúrgico es el tratamiento conservador hasta el parto o hasta confirmar la progresión del tumor (47-50).

Tumores del estroma gastrointestinal

Son tumores raros (frecuencia de 1 caso por 100000 pacientes/año y representan $0,1 \%-3 \%$ de las neoplasias gastrointestinales) que pertenecen a la familia de los sarcomas de tejidos blandos. Afectan al tejido conectivo $\mathrm{y}$, como este tejido se encuentra diseminado, pueden aparecer en casi cualquier lugar. Con mayor frecuencia están presentes en estómago $(60 \%-80 \%)$, duodeno e intestino delgado $(20 \%-30 \%)$ (51-53). En una revisión de 12 casos en embarazadas, se encontró enfermedad metastásica en bazo, hígado, pulmones y peritoneo en $30 \%$ de los casos. Once pacientes fueron sometidos a cirugía para resección tumoral primaria. La edad gestacional promedio al momento del parto fue de 36 semanas. No se encontraron metástasis placentarias o fetales (54).

Los síntomas clínicos más comunes son: molestias abdominales, sensación de saciedad, náuseas, vómitos, sangrado gastrointestinal, anemia y debilidad generalizada (55). El diagnóstico por imágenes durante el embarazo se realiza por ecografía y resonancia magnética (después del primer trimestre). El diagnóstico final es realizado por el examen histopatológico con determinación de la expresión de CD117 y CD34. Los factores pronósticos más importantes son: la ubicación del tumor (mejor pronóstico si la localización es el estómago), el tamaño del tumor (mayor de 5 centímetros - peor pronóstico), el número de mitosis (mayores de 5/50 grandes campos de visión - peor pronóstico), las mutaciones en el gen KIT (peor pronóstico) y PDGFRA (la mutación del exón 18 está asociada con mejor pronóstico) (54-57).

La cirugía radical es el tratamiento estándar para tumores localizados. Es recomendable la interrupción del embarazo a las 35 - 37 semanas en aquellos casos diagnosticados en la segunda mitad del embarazo, para luego realizar el tratamiento quirúrgico y terapia sistémica con imatinib. En situaciones de riesgo materno, complicaciones significativas, alta dinámica de la enfermedad y diagnóstico en la primera mitad del embarazo, puede considerarse la interrupción del embarazo y luego el tratamiento estándar utilizado en la población general. La resección tumoral también puede realizarse, preferiblemente, después del primer trimestre del embarazo y el tratamiento sistémico (imatinib) debe iniciarse solo después del parto. Una alternativa al tratamiento quirúrgico puede ser el tratamiento sintomático hasta el parto o confirmación de la progresión del tumor local. La forma óptima de interrupción del embarazo parece ser la cesárea (54).

El tratamiento sistémico con imatinib durante el embarazo se asocia con alto riesgo de defectos fetales y aborto espontáneo. Solo $50 \%$ de las pacientes tratadas presentaron parto normal, la mitad restante terminó en aborto espontáneo. Sin embargo, debido a la falta de datos y de observaciones, no se recomienda su uso durante el embarazo $(54,57,58)$. 


\section{Tumores hepáticos}

También son muy raros durante el embarazo. Desde 1950 se han descrito alrededor de 50 casos en el mundo (59). Los síntomas en casos de carcinoma hepatocelular son similares a los síntomas del embarazo (náuseas, vómitos y fatiga), lo que lleva a retraso diagnóstico. En embarazadas, existe un aumento en las concentraciones de alfafetoproteína (las concentraciones disminuyen luego de las 30 semanas de gestación para normalizarse aproximadamente 2 semanas después del parto), por lo que no es útil en el diagnóstico. La ecografía puede suministrar elementos de sospecha diagnóstica, pero las imágenes de resonancia magnética confirman el diagnóstico. Los métodos para tratar el cáncer de hígado incluyen: tratamiento quirúrgico, quimioembolización arterial, termoablación, radioembolización y quimioterapia sistémica $(60,61)$.

Existen estudios de series de casos que demuestran que la tasa de supervivencia luego de la resección tumoral a los 6 meses, 1 , 2 y 3 años son de $50 \%, 30 \%$, $18 \%$ y $14 \%$, respectivamente. El tiempo de supervivencia promedio en este grupo de pacientes parece ser más corto, comparado con la población general. Esto puede mejorarse con el diagnóstico en etapas más tempranas y tratamiento quirúrgico efectivo $(62,63)$.

Cuando el cáncer de hígado es diagnosticado durante el primer trimestre del embarazo, se recomienda la interrupción de este e iniciar un régimen terapéutico oncológico con estándares similares a la población general. Debido al mal pronóstico, no es recomendable retrasar el tratamiento. Durante el segundo trimestre, la anestesia general y resección hepática parecen ser seguras para el feto, por lo tanto, es posible continuar el embarazo, aunque algunos autores recomiendan interrumpirlo si el diagnóstico es antes de las 28 semanas. La solución óptima es el manejo individualizado dependiendo de la decisión materna, de las posibilidades técnicas del tratamiento quirúrgico y de factores relacionados al embarazo. Si el diagnóstico es durante el tercer trimestre, se debe posponer la cirugía hasta la interrupción quirúrgica del embarazo, momento en el cual debe realizarse simultáneamente la resección del tumor. Aproximadamente, $10 \%$ de los casos tienen complicaciones como ruptura tumoral y hemorragia. La resección del tumor es un procedimiento que puede salvar la vida de la madre $(63,64)$.

\section{CONCLUSIÓN}

Tanto el procedimiento diagnóstico y manejo terapéutico, como la atención médica y decisión de la interrupción del embarazo, deben individualizarse y discutirse entre el equipo multidisciplinario. El manejo depende de factores relacionados al cáncer (tipo, ubicación y estadio clínico), factores maternos (estado general, comorbilidades, potenciales complicaciones y deseo de la madre) y factores relacionados al embarazo (edad gestacional y posibilidad de obtener un recién nacido sano). Las posibilidades y procedimientos terapéuticos deben discutirse con la paciente $\mathrm{y}$ su familia para tomar una decisión final sobre los procedimientos obstétricos y oncológicos adecuados

\section{REFERENCIAS}

1. Pavlidis N. Cancer and pregnancy: what should we know about the management with systemic treatment of pregnant women with cancer? Eur J Cancer. 2011; 47 Suppl 3:S348-352. doi: 10.1016/S09598049(11)70199-X.

2. Papathanasiou K, Tolikas A, Savvidis A, Traianos V, Tzafettas J.Advanced sigmoid colon cancer in pregnancy presented as an abscess. J Obstet Gynaecol. 2004; 24(3):319-320. doi: 10.1080/01443610410001661020.

3. Lui MW, Lai CW, Ong CY. Metastatic pancreatic adenocarcinoma presented as back pain in pregnancy: case report and review of literature. J Matern Fetal Neonatal Med. 2016; 29(9):1421-1424. doi: $10.3109 / 14767058.2015 .1049942$. 


\section{E REYNA-VILLASMIL}

4. Song MJ, Park YS, Song HJ, Park SJ, Ahn JY, Choi $\mathrm{KD}$, et al. Prognosis of Pregnancy-Associated Gastric Cancer: An Age-, Sex-, and Stage-Matched CaseControl Study. Gut Liver. 2016; 10(5):731-738. doi: 10.5009/gnl15323.

5. Shim MH, Mok CW, Chang KH, Sung JH, Choi SJ, Oh SY, et al. Clinical characteristics and outcome of cancer diagnosed during pregnancy. Obstet Gynecol Sci. 2016; 59(1):1-8. doi: 10.5468/ogs.2016.59.1.1.

6. Al-Ibrahim A, Parrish J, Dunn E, Swallow C, Maxwell

C. Pregnancy and maternal outcomes in women with prior or current gastrointestinal malignancies. J Obstet Gynaecol Can. 2014; 36(1):34-41. doi: 10.1016/S17012163(15)30681-2.

7. Perazzo R, Pinto L, Quijada O, Galvis E, Bethelmy A. Cáncer de esófago, experiencia de 24 años, Hospital "Dr. Miguel Pérez Carreño" años 1980-2004. GEN. 2006; 60 (4): 279-283.

8. Al-Githmi I. Esophageal cancer associated with pregnancy. J Obstet Gynaecol Can. 2009; 31(8):730731. doi: 10.1016/S1701-2163(16)34277-3.

9. Şahin M, Kocaman G, Özkan M, Yüksel C, Enön S, Kutlay H. Resection of esophageal carcinoma during pregnancy. Ann Thorac Surg. 2015; 99(1):333-335. doi: 10.1016/j.athoracsur.2014.01.080.

10. Sakamoto K, Kanda T, Ohashi M, Kurabayashi T, Serikawa $\mathrm{T}$, Matsunaga $\mathrm{M}$, et al. Management of patients with pregnancy-associated gastric cancer in Japan: a mini-review. Int J Clin Oncol. 2009; 14(5):392396. doi: 10.1007/s10147-009-0903-6.

11. Lee HJ, Lee IK, Kim JW, Lee KU, Choe KJ, Yang HK. Clinical characteristics of gastric cancer associated with pregnancy. Dig Surg. 2009; 26(1):31-36. doi: 10.1159/000193330.

12. Bacic B, Misic Z, Kovacic V, Sprung J. Gastric carcinoma presenting as pericardial tamponade during pregnancy. Turk J Gastroenterol. 2009; 20(4):276-278. doi: 10.4318/tjg.2009.0026.

13. Whittington JR, Whitcombe DD, Eads LE, Jeffus SK, Quick CM, Wendel PJ, et al. Signet Ring Cell Carcinoma with Lymphangitic Carcinomatosis in Pregnancy: A Case Report of an Unexpected Maternal Death and Review of the Literature. Am J Case Rep. 2019; 20:1888-1891. doi: 10.12659/AJCR.919412.

14. Jeong B, Shim JY, Kim CJ, Won HS, Lee PR, Kim A. Massive perivillous fibrin deposition in the placenta and uterine metastasis of gastric adenocarcinoma during pregnancy. J Obstet Gynaecol Res. 2014; 40(4):11501153. doi: 10.1111/jog.12292.
15. Moro B, Moro J, Gelder O, Mendoza F, García G. Cáncer gástrico precoz: reporte de un caso. Rev Venez Oncol. 2002; 24 (1): 61-63.

16. Fill S, Taran A, Schulz HU, Kahl S, Kalinski T, Smith B, et al. Sister Mary Joseph's nodule as the first sign of pregnancy-associated gastric cancer: a case report. World J Gastroenterol. 2008; 14(6):951-953. doi: 10.3748/wjg. 14.951.

17. Takai H, Kondoh E, Sato Y, Kakui K, Tatsumi K, Konishi I. Disseminated intravascular coagulation as the presenting sign of gastric cancer during pregnancy. J Obstet Gynaecol Res. 2011; 37(11):1717-1719. doi: 10.1111/j.1447-0756.2011.01561.x.

18. Nishie H, Mizushima T, Suzuki Y, Fukusada S, Inoue $\mathrm{T}$, Kachi $\mathrm{K}$, et al. Chemotherapy treatment of a pregnant woman with progressive gastric cancer. Intern Med. 2015; 54(10):1207-1212. doi: 10.2169/ internalmedicine.54.3973.

19. Hepner A, Negrini D, Hase EA, Exman P, Testa L, Trinconi AF, et al. Cancer During Pregnancy: The Oncologist Overview. World J Oncol. 2019; 10(1):2834. doi: 10.14740/wjon 1177.

20. Eastwood-Wilshere N, Turner J, Oliveira N, Morton A. Cancer in Pregnancy. Asia Pac J Clin Oncol. 2019; 15(6):296-308. doi: 10.1111/ajco.13235.

21. Ning S, Yang Y, Wang C, Luo F. Pseudomyxoma peritonei induced by low-grade appendiceal mucinous neoplasm accompanied by rectal cancer: a case report and literature review. BMC Surg. 2019; 19(1):42. doi: 10.1186/s12893-019-0508-6.

22. Lu YY, Guo AT, Liu AJ, Shi HY. [Prognostic value of clinical and pathological characteristics in 48 women with pseudomyxoma peritonei]. Zhonghua $\mathrm{Fu}$ Chan $\mathrm{Ke}$ Za Zhi. 2013; 48(8):595-601. Chinese. DOI: 10.3760/ cma.j.issn.0529-567x.2013.08.008

23. Haase E, Yoo D, Sugarbaker PH. Management of appendiceal pseudomyxoma peritonei diagnosed during pregnancy. World J Surg Oncol. 2009; 7:48. doi: 10.1186/1477-7819-7-48.

24. Koyama S, Tomimatsu T, Sawada K, Kanagawa T, Tsutsui T, Kimura T. Pseudomyxoma peritonei originating from colorectal cancer during pregnancy. J Obstet Gynaecol Res. 2011; 37(3):254-258. doi: 10.1111/j.1447-0756.2010.01379.x.

25. Sugarbaker PH. Peritoneum as the first-line of defense in carcinomatosis. J Surg Oncol. 2007; 95(2):93-96. doi: 10.1002/jso.20676. PMID: 17262739

26. Berrueta L, Salmen S, Montes H, Hernández M. Historia natural del carcinoma de colon y recto. GEN. 1999; 53 (1): 45-53. 
27. Kömürcü S, Ozet A, Oztürk B, Arpaci F, Altundağ MK, Tezcan Y. Colon cancer during pregnancy. A case report. J Reprod Med [Internet]. 2001 [consultado enero de 2020]; 46(1):75-8. Disponible en: https:// europepmc.org/article/med/11209639

28. Capriglione S, Manzano A, Gulino FA, Lopez S. Management of gynecological cancer in pregnant patients. Minerva Ginecol. 2019; 71(2):133-145. doi: 10.23736/S0026-4784.18.04324-1.

29. Walsh C, Fazio VW. Cancer of the colon, rectum, and anus during pregnancy. The surgeon's perspective. Gastroenterol Clin North Am. 1998; 27(1):257-267. doi: 10.1016/s0889-8553(05)70356-3.

30. Dahling MT, Xing G, Cress R, Danielsen B, Smith LH. Pregnancy-associated colon and rectal cancer: perinatal and canceroutcomes. JMaternFetal Neonatal Med.2009; 22(3):204-211. doi: 10.1080/14767050802559111.

31. Haggar F, Pereira G, Preen D, Woods J, Martel G, Boushey $\mathrm{R}$, et al. Maternal and neonatal outcomes in pregnancies following colorectal cancer. Surg Endosc. 2013; 27(7):2327-2336. doi: 10.1007/s00464-0122774-6.

32. Yaghoobi M, Koren G, Nulman I. Challenges to diagnosing colorectal cancer during pregnancy. Can Fam Physician [Internet]. 2009 [consultado enero de 2020]; 55(9):881-885. Disponible en: https://www.cfp. $\mathrm{ca} /$ content $/ 55 / 9 / 881$.long

33. Toosi M, Moaddabshoar L, Malek-Hosseini SA, Sasani MR, Mokhtari M, Mohammadianpanah M. Rectal cancer in pregnancy: a diagnostic and therapeutic challenge. J Egypt Natl Canc Inst. 2014; 26(3):175179. doi: 10.1016/j.jnci.2014.03.003.

34. Salani R, Billingsley CC, Crafton SM. Cancer and pregnancy: an overview for obstetricians and gynecologists. Am J Obstet Gynecol. 2014; 211(1):714. doi: 10.1016/j.ajog.2013.12.002.

35. Chan YM, Ngai SW, Lao TT. Colon cancer in pregnancy. A case report. J Reprod Med [Internet]. 1999 [consultado enero de 2020]; 44(8):733-736. Disponible en: https://pubmed.ncbi.nlm.nih.gov/10483546/

36. Gensheimer M, Jones CA, Graves CR, Merchant NB, Lockhart AC. Administration of oxaliplatin to a pregnant woman with rectal cancer. Cancer Chemother Pharmacol. 2009; 63(2):371-373. doi: 10.1007/s00280008-0731-9.

37. Kanate AS, Auber ML, Higa GM. Priorities and uncertainties of administering chemotherapy in a pregnant woman with newly diagnosed colorectal cancer. J Oncol Pharm Pract. 2009; 15(1):5-8. doi: $10.1177 / 1078155208094101$.
38. Jeppesen JB, Østerlind K. Successful twin pregnancy outcome after in utero exposure to FOLFOX for metastatic colon cancer: a case report and review of the literature. Clin Colorectal Cancer. 2011; 10(4):348352. doi: 10.1016/j.clcc.2011.06.003.

39. Makoshi Z, Perrott C, Al-Khatani K, Al-Mohaisen F. Chemotherapeutic treatment of colorectal cancer in pregnancy: case report. J Med Case Rep. 2015; 9:140. doi: 10.1186/s13256-015-0621-9.

40. Cirillo M, Musola M, Cassandrini PA, Lunardi G, Venturini M. Irinotecan during pregnancy in metastatic colon cancer. Tumori. 2012; 98(6):155e-157e. doi: 10.1700/1217.13511.

41. Taylor J, Amanze A, Di Federico E, Verschraegen C. Irinotecan use during pregnancy. Obstet Gynecol. 2009; 114(2 Pt 2):451-452. doi: 10.1097/ AOG.0b013e3181a1d478.

42. Wiseman JE, Yamamoto M, Nguyen TD, Bonadio J, Imagawa DK. Cystic pancreatic neoplasm in pregnancy: a case report and review of the literature. Arch Surg. 2008; 143(1):84-86. doi: 10.1001/archsurg.2007.4.

43. Shirakawa S, Matsumoto I, Nakayama S, Mukubo $\mathrm{H}$, Toyama H, Shinzeki M, et al. Mucinous cystic neoplasm of the pancreas associated with pregnancy: report of two cases. Nihon Shokakibyo Gakkai Zasshi. 2010; 107(11):1828-1834. https://doi.org/10.11405/ nisshoshi.107.1828

44. Kanji ZS, Gallinger S. Diagnosis and management of pancreatic cancer. CMAJ. 2013; 185(14):1219-1226. doi: 10.1503/cmaj.121368.

45. Christiansen E, Vestergaard $H$. Insulinoma in a third-trimester pregnant woman combined with preeclampsia: a case report and review of the diagnostic strategies. Gynecol Endocrinol. 2008; 24(7):417-422. doi: 10.1080/09513590802210931.

46. Rodríguez Y, Gordils A, Hidalgo D, Petit O, Galeno L, González J. Tumor carcinoide apendicular: reporte de un caso. Centro Méd. 2002; 47 (1): 65-67.

47. Pitiakoudis M, Kirmanidis M, Tsaroucha A, Christianakis E, Filippou D, Sivridis E, et al. Carcinoid tumor of the appendix during pregnancy. A rare case and a review of the literature. J BUON. 2008; 13(2):271275. Disponible en: https://www.researchgate.net/ publication/5300237_Carcinoid_tumor_of_the appendix_during_pregnancy_A_rare_case_and_a review_of_the_literature

48. Crha K, Novák M, Lenz J. Carcinoid of the appendix during pregnancy. Ceska Gynekol. Spring [Internet] 2017 [consultado enero 2020]; 82(2):126-128. Disponible en: https://www.prolekare.cz/en/journals/ 
czech-gynaecology/archive/2017-2-13

49. Shapiro R, Eldar S, Sadot E, Papa MZ, Zippel DB. Appendiceal carcinoid at a large tertiary center: pathologic findings and long-term follow-up evaluation. Am J Surg. 2011; 201(6):805-808. doi: 10.1016/j. amjsurg.2010.04.016.

50. Louzi A, Nargis Y, Belaabidia B, Alatawna H, Benelkhaiat R, Finech B, et al. [Appendicular mucocele caused by carcinoid tumor during pregnancy]. Gynecol Obstet Fertil. 2006; 34(11):1051-1054. French. doi: 10.1016/j.gyobfe.2006.07.037.

51. Cao H, Zhang Y, Wang M, Shen DP, Sheng ZY, $\mathrm{Ni} \mathrm{XZ}$, et al. Prognostic analysis of patients with gastrointestinal stromal tumors: a single unit experience with surgical treatment of primary disease. Chin Med J (Engl). 2010; 123(2):131-136. doi: 10.3760/cma.j.is sn.0366-6999.2010.02.001

52. Miettinen M, Majidi M, Lasota J. Pathology and diagnostic criteria of gastrointestinal stromal tumors (GISTs): a review. Eur J Cancer. 2002; 38 Suppl 5:S3951. doi: 10.1016/s0959-8049(02)80602-5.

53. Acevedo J, Moreno E, Rodríguez JJ, Lión L, Márquez G. Tumores del estroma gastrointestinal: resultados del tratamiento. Rev Venez Oncol. 2006; 18(1):14-18.

54. Zarkavelis G, Petrakis D, Pavlidis N. Gastrointestinal stromal tumors during pregnancy: a systematic review of an uncommon but treatable malignancy. Clin Transl Oncol. 2015; 17(10):757-762. doi: 10.1007/s12094015-1315-x.

55. Charville GW, Longacre TA. Surgical Pathology of Gastrointestinal Stromal Tumors: Practical Implications of Morphologic and Molecular Heterogeneity for Precision Medicine. Adv Anat Pathol. 2017; 24(6):336353. doi: 10.1097/PAP.0000000000000166.

56. Rubin BP, Blanke CD, Demetri GD, Dematteo RP, Fletcher CD, Goldblum JR, et al.; Cancer Committee, College of American Pathologists. Protocol for the examination of specimens from patients with gastrointestinal stromal tumor. Arch Pathol Lab Med. 2010; 134(2):165-170. doi: 10.5858/134.2.165.

57. Goel N, Malik R, Rathi B, Bhaskaran S, Rajaram S, Mehta S, etal.Pregnancy with metastatic gastrointestinal stromal tumor (GIST) on imatinib chemotherapy: an oncologist's nightmare and obstetrician's dilemma. J Gastrointest Cancer. 2013; 44(1):115-117. doi: 10.1007/s12029-012-9464-4.
58. Pye SM, Cortes J, Ault P, Hatfield A, Kantarjian H, Pilot $\mathrm{R}$, et al. The effects of imatinib on pregnancy outcome. Blood. 2008; 111(12):5505-5508. doi: 10.1182/blood-2007-10-114900.

59. Chen HW, Li JY, Huang PQ, Chen RF, Lai EC, Lau WY. Synchronous right hepatectomy and cesarean section in a pregnant lady with hepatocellular carcinoma. Int J Surg Case Rep. 2013; 4(1):112-114. doi: 10.1016/j. ijscr.2012.10.014.

60. Alvarez de la Rosa M, Nicolás-Pérez D, Muñiz-Montes JR, Trujillo-Carrillo JL. Evolution and management of a hepatocellular carcinoma during pregnancy. J Obstet Gynaecol Res. 2006; 32(4):437-9. doi: 10.1111/j.14470756.2006.00421.x.

61. Cobey FC, Salem RR. A review of liver masses in pregnancy and a proposed algorithm for their diagnosis and management. Am J Surg. 2004; 187(2):181-191. doi: 10.1016/j.amjsurg.2003.11.016.

62. Choi KK, Hong YJ, Choi SB, Park YN, Choi JS, Lee $\mathrm{WJ}$, et al. Hepatocellular carcinoma during pregnancy: is hepatocellular carcinoma more aggressive in pregnant patients? J Hepatobiliary Pancreat Sci. 2011; 18(3):422-431. doi: 10.1007/s00534-010-0345-6.

63. Matsuo M, Furukawa K, Shimizu H, Yoshitomi H, Takayashiki T, Kuboki S, et al. Novel treatment strategy with radiofrequency ablation and surgery for pregnant patients with hepatocellular carcinoma: a case report. Surg Case Rep. 2018; 4(1):43. doi: 10.1186/s40792018-0434-3.

64. Li AJ, Zhou WP, Wu MC, Luo XJ. Hepatectomy after primary repair of ruptured liver cancer. Hepatobiliary Pancreat Dis Int [Internet]. 2007 [consultado enero de 2020]; 6(3):267-270. Disponible en: https://pubmed. ncbi.nlm.nih.gov/17548249/

Recibido 19 de febrero de 2020 Aprobado 15 de mayo de 2020 